Acta Botanica Brasilica - 30(3): 407-421. July-September 2016. doi: 10.1590/0102-33062016abb0074

\title{
Root fungal associations in some non-orchidaceous vascular lithophytes
}

\author{
Thangavelu Muthukumar ${ }^{1 *}$, Marimuthu Chinnathambi' and Perumalsamy Priyadharsini'
}

Received: March 7, 2016

Accepted: July 11, 2016

\begin{abstract}
Plant roots in natural ecosystems are colonized by a diverse group of fungi among which the most common and widespread are arbuscular mycorrhizal (AM) and dark septate endophyte (DSE) fungi. Though AM and DSE fungal associations are well reported for terricolous plant species, they are rather poorly known for lithophytic plant species. In this study, we examined AM and DSE fungal association in 72 non-orchidaceous vascular plant species growing as lithophytes in Siruvani Hills, Western Ghats of Tamilnadu, India. Sixty-nine plant species had AM and 58 species had DSE fungal associations. To our knowledge, we report AM fungal association in 42 and DSE fungal association in 53 plant species for the first time. There were significant differences in total root length colonization and root length colonized by different AM and DSE fungal structures among plant species. In contrast, the differences in AM and DSE fungal colonization among plants in various life-forms and lifecycles were not significant. AM morphology reported for the first time in 56 plant species was dominated by intermediate type AM morphology. A significant negative relationship existed between total root length colonized by AM and DSE fungi. These results clearly suggest that AM and DSE fungal associations are widespread in lithophytes.
\end{abstract}

Keywords: arbuscular mycorrhizal fungi, dark septate endophyte fungi, life cycle, life-forms, microsclerotia

\section{Introduction}

Plants grow in a variety of habitats, including those which are not normally suitable for plant growth. One of the harshest habitats is the surfaces and crevices of rock where low moisture, nutrients and high temperatures limit plant's existence. Plants growing on rocks are termed lithophytes and these types of plants occur in different arid (Zwieniecki \& Newton 1995; Bashan et al. 2002; Lopez et al. 2009) and non arid (Tozer et al. 2005; Gröger \& Huber 2007; Muthuraja et al. 2014) ecosystems. Nevertheless, lithophytes also colonize rocks that are constantly misted by waterfalls or rocks dripping with water. Lithophytes have certain biological peculiarities, including capturing moisture from rain, dew, melting snow or water trickling down the rocks, and acquire nutrients from rain water, litter, and feeding on insects or their own dead tissues (Alves \& Kolbeck 1993; Gold \& Bliss 1995; Tozer et al. 2005). These features enable lithophytes to occupy and thrive on the rock surfaces. Based on their existence and dependence on rock, lithophytes are classified as epilithic or epipetric and endolithic forms. Epilithic forms grow superficially on rock surfaces (e.g., orchids, ferns, algae, bryophytes), whereas endolithic forms grow embedded in rocks (e.g., algae, fungi). Further, plants growing exclusively on rocks are known as obligate lithophytes and those that growing

\footnotetext{
${ }^{1}$ Root and Soil Biology Laboratory, Department of Botany, Bharathiar University, Coimbatore 641046, Tamilnadu, India

*Corresponding author: tmkum@yahoo.com
} 
on rocky as well as on other substrates are known as the facultative lithophytes.

Generally, success of plants growing on harsh environment depends on their ability to associate with microorganisms (Puente et al. 2004a; b; Bashan et al. 2007). Plants growing in different environments are colonized by arbuscular mycorrhizal (AM) fungi belonging to Glomeromycota, and it is one of the most widespread associations among land plants (Smith \& Read 2008). The AM fungi aids plants in their uptake of water and nutrients, and impart tolerance against various stresses. This association enables plant's to survive and thrive under harsh environments that are not conducive for plant growth. Although the roots of several terricolous plant taxa have been examined for the presence of AM fungal association, their occurrence in lithophytic or saxicolous plant species is limited to certain plant groups. For example, the occurrence of AM fungal association has been examined in lithophytic mosses (Zhang \& Guo 2007) and ferns (Lehnert et al. 2009; Kessler et al. 2010; Muthukumar \& Prabha 2013; Muthuraja et al. 2014). The results of some of these studies suggest that non-mycorrhizal condition appears to be more prevalent in lithophytic than in terricolous taxa (Kessler et al. 2010).

A great diversity exists in inter- and intracellular distribution of AM fungal structures within plant roots. Based on the distribution of the fungal structures within plant roots, the AM colonization patterns have been termed as Arum-, Paris- and intermediate types. The intraradical fungal hyphae are linear and intercellular in Arum-type, and intracellular and coiled in Paris-type. In between these two extremes, there exists a diverse colonization patterns termed as intermediate types. Though AM colonization patterns in terricolous plant species are widely reported, it is rather scanty for lithophytic species (e.g., Muthukumar \& Prabha 2013; Muthuraja et al. 2014).

A group of predominantly ascomycetous fungi belonging to the order Helotiales also colonizes plant roots either individually or along with AM fungi. These fungi termed as dark septate endophyte (DSE) fungi form dematiaceous, regularly septate hyphae with microsclerotia and/or moniliform cells in plant roots. Although these fungi have been recorded in roots of more than 1000 plant species growing in a range of habitats (Jumpponen \& Trappe 1998; Mandyam \& Jumpponen 2005; Muthukumar \& Prabha 2013; Muthuraja et al. 2014; Kumar \& Muthukumar 2014), their occurrences in many plant taxa and habitats are yet to be examined. Like mycorrhizal fungi, DSE fungi also associate with plants under extreme environmental conditions (Read \& Haselwandter 1981; Newsham et al. 2009). The widespread occurrence of DSE fungi in stressed environments suggests that DSE fungi might act as surrogate mutualists in these environments (Bledsoe et al. 1990; Newsham 2011). Inspite of their frequent occurrence in plant roots, the role of DSE fungi on plant growth, development and ecology is largely unknown. Limited studies exploring the role of DSE fungi on plant growth show that these fungi could enhance plant performance when nutrients are available predominantly in organic forms (Newsham 2011). Like the incidence of AM, DSE fungal associations in lithophytes are also limited to certain plant groups. The occurrence of DSE fungal association in lithophytic fern and fern-allies has been reported recently from the Eastern and Western Ghats of southern India (Muthukumar et al. 2014; Muthuraja et al. 2014).

As lithophytic habitats are characterized by extreme environmental conditions and plants in stressed habitats depend more on association with endophytic microorganisms for their growth and survival, we hypothesized that the incidence of endophytic root fungal associations in lithophytes are high. To test this hypothesis, we examined the roots of non-orchidaceous lithophytes to assess the incidence and extent of AM and DSE fungal colonization. In addition, we assessed the influence of plant habit and life cycle on the occurrence of AM and DSE fungal associations. Finally, we also determined the existence of any possible relationship between the AM and DSE fungal variables as these fungal types occupy the same niche within plant roots.

\section{Materials and methods}

\section{Sampling site and investigated plant species}

Kovai Kutralam ( $\left.10^{\circ} 56^{\prime} 12.9^{\prime \prime} \mathrm{N} 76^{\circ} 41^{\prime} 14.3^{\prime \prime} \mathrm{E}\right)$, located 22 miles West of Coimbatore City in Siruvani Hills is a part of the Nilgiri Biosphere Reserve of Western Ghats, which is one of the biodiversity hotspots of the world (Balaji \& Hariharan 2013). In addition to providing drinking water to the Coimbatore city, Siruvani Hills are also home to certain tribes like the Mudugars and Irulars. The vegetation at lower to higher altitudes ranges from southern dry mixed deciduous forest, southern moist mixed deciduous forest to tropical evergreen forest in hill tops (Balaji \& Hariharan 2013). This region receives a rainfall varying from 750 to $2000 \mathrm{~mm}$, mostly from the northeast monsoon during September to November, with a dry period from December to May. The minimum and maximum temperature range from 10 to $22{ }^{\circ} \mathrm{C}$ (average $16{ }^{\circ} \mathrm{C}$ ) and 35 to $43{ }^{\circ} \mathrm{C}$ (average $39^{\circ} \mathrm{C}$ ) respectively (Balaji \& Hariharan 2013). The 72 nonorchidaceous plant species in this study belong to 65 genera and 38 families (Tab. 1). Twelve of these were pteridophytes belonging to seven families. Fifty-five plant species were herbs, 14 were shrubs/under shrubs, and three were trees. Seventeen species were annuals, and 55 were perennials (Tab. 1). Plant nomenclature follows The Plant list (www. ipni.org).

\section{Sample collection}

All the root samples were taken between December 2009 and February 2010. Three plants were sampled for 
Table 1. Life-form, life cycle, arbuscular mycorrhizal (AM) and dark septate endophyte (DSE) fungal status, AM morphology (in parenthesis) of non-orchidaceous lithophytes in Siruvani Hills of Western Ghats. In the last column, previous reports on the occurrence of AM and DSE fungal associations and AM morphological types (in parenthesis) are listed.

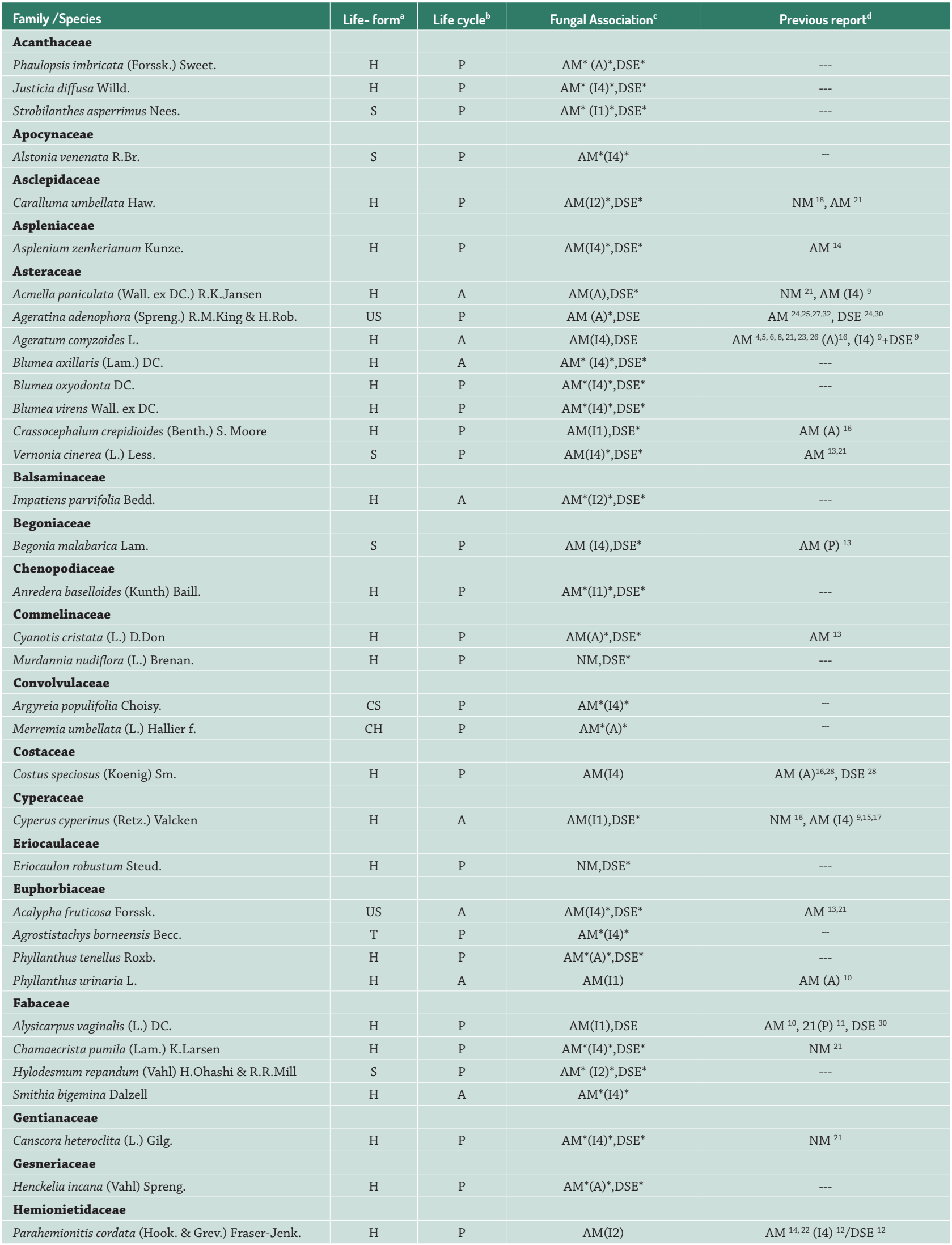


Thangavelu Muthukumar, Marimuthu Chinnathambi and Perumalsamy Priyadharsini

Table 1. Cont.

\begin{tabular}{|c|c|c|c|c|}
\hline Family/Species & Life- form ${ }^{a}$ & Life $_{\text {cycle }}{ }^{b}$ & Fungal Association ${ }^{c}$ & Previous report ${ }^{d}$ \\
\hline \multicolumn{5}{|l|}{ Lamiaceae } \\
\hline Anisochilus suffruticosus Wight. & US & $\mathrm{P}$ & $\mathrm{AM}^{*}(\mathrm{~A})^{*}, \mathrm{DSE}^{*}$ & --- \\
\hline Plectranthus amboinicus (Lour.) Spreng. & S & $\mathrm{P}$ & $\operatorname{AM}^{*}(\mathrm{~A})^{*}, \mathrm{DSE}^{*}$ & - \\
\hline Pogostemon plectrantoides Desf. & s & $\mathrm{P}$ & $\mathrm{AM}^{*}(\mathrm{I} 1)^{*}, \mathrm{DSE}^{*}$ & - \\
\hline \multicolumn{5}{|l|}{ Lentibulariaceae } \\
\hline Utricularia uliginosa Vahl & $\mathrm{H}$ & A & $\mathrm{AM}^{*}(\mathrm{~A})^{*}, \mathrm{DSE}^{*}$ & $\mathrm{NM}^{13}$ \\
\hline \multicolumn{5}{|l|}{ Liliaceae } \\
\hline Sansevieria zeylanica (L.) Willd. & $\mathrm{H}$ & $\mathrm{P}$ & $\mathrm{AM}^{*}(\mathrm{I} 1)^{*}, \mathrm{DSE}^{*}$ & - \\
\hline \multicolumn{5}{|l|}{ Lygodiaceae } \\
\hline Lygodium flexuosum (L.) Sw. & $\mathrm{H}$ & $\mathrm{P}$ & $\mathrm{AM}^{*}(\mathrm{I} 2)^{*}$ & $\mathrm{NM}^{7}$ \\
\hline \multicolumn{5}{|l|}{ Malvaceae } \\
\hline Hibiscus surattensis $\mathrm{L}$. & US & $\mathrm{P}$ & $\mathrm{AM}(\mathrm{I} 4)^{*}, \mathrm{DSE}^{*}$ & $\mathrm{AM}^{21}$ \\
\hline \multicolumn{5}{|l|}{ Melastomataceae } \\
\hline Osbeckia wynaadensis C.B. Clarke & S & $\mathrm{P}$ & $\mathrm{AM}^{*}(\mathrm{~A})^{*}, \mathrm{DSE}^{*}$ & - \\
\hline \multicolumn{5}{|l|}{ Meliaceae } \\
\hline Melia azedarach L. & $\mathrm{T}$ & $\mathrm{P}$ & $\mathrm{AM}(\mathrm{II})^{*}, \mathrm{DSE}^{*}$ & $\mathrm{NM}^{13}, \mathrm{AM}^{16}$ \\
\hline \multicolumn{5}{|l|}{ Moraceae } \\
\hline Ficus microcarpa L.f. & $\mathrm{T}$ & $P$ & $\mathrm{AM}^{*}(\mathrm{I} 4)^{*}, \mathrm{DSE}^{*}$ & $\mathrm{NM}^{21}$ \\
\hline \multicolumn{5}{|l|}{ Nephrolepidaceae } \\
\hline Nephrolepis cordifolia (L.) C. Presl & $\mathrm{H}$ & A & $\mathrm{AM}(\mathrm{P})^{*}, \mathrm{DSE}$ & $\mathrm{AM}^{1},{ }^{12},{ }^{14},{ }^{22}, \mathrm{DSE}^{12}$ \\
\hline \multicolumn{5}{|l|}{ Oxalidaceae } \\
\hline Biophytum sensitivum (L.) DC. & $\mathrm{H}$ & A & $\mathrm{AM}^{*}(\mathrm{I} 4)^{*}, \mathrm{DSE}^{*}$ & $\mathrm{NM}^{21}$ \\
\hline \multicolumn{5}{|l|}{ Poaceae } \\
\hline Dimeria kanijirapallilana K.C.Jacob. & $\mathrm{H}$ & $\mathrm{P}$ & $\mathrm{AM}^{*}(\mathrm{I} 4)^{*}, \mathrm{DSE}^{*}$ & - \\
\hline Eragrostis atrovirens (Desf.) Trin.ex Steud. & $\mathrm{H}$ & $\mathrm{P}$ & $\mathrm{AM}^{*}(\mathrm{I} 4)^{*}, \mathrm{DSE}^{*}$ & --- \\
\hline Garnotia tenella (Arn. ex Miq.) Janowski & $\mathrm{H}$ & A & $\mathrm{AM}^{*}(\mathrm{I} 4)^{*}, \mathrm{DSE}^{*}$ & - \\
\hline Oplismenus burmanni (Retz.) P.Beauv. & $\mathrm{H}$ & A & $\mathrm{AM}(\mathrm{I} 4)^{*}$ & $\mathrm{AM}^{21}$ \\
\hline Oplismenus compositus (L.) P. Beauv. & $\mathrm{H}$ & $\mathrm{P}$ & $\mathrm{AM}(\mathrm{I} 4), \mathrm{DSE}^{*}$ & $\mathrm{AM}(\mathrm{P})^{13}$ \\
\hline Pogonatherum crinitum (Thunb.) Kunth & $\mathrm{H}$ & $\mathrm{P}$ & $\mathrm{AM}(\mathrm{I} 4), \mathrm{DSE} \mathrm{E}^{*}$ & $\mathrm{AM}^{29}(\mathrm{P})^{13}$ \\
\hline Setaria verticillata (L.) P. Beauv. & $\mathrm{H}$ & A & $\mathrm{AM}(\mathrm{I} 4)^{*}$ & $\mathrm{AM}^{2,3}, \mathrm{DSE}^{20}$ \\
\hline \multicolumn{5}{|l|}{ Polypodiaceae } \\
\hline Drynaria quercifolia (L.) J. Sm. & $\mathrm{H}$ & $\mathrm{P}$ & NM & $\mathrm{NM}^{14}, \mathrm{AM}^{22}(\mathrm{P} / \mathrm{I})^{19}(\mathrm{I} 4)^{12}, \mathrm{DSE}^{19}$ \\
\hline Pleopeltis macrocarpa (Bory ex Willd.) Kaulf. & $\mathrm{H}$ & $\mathrm{P}$ & $\mathrm{AM}^{*}(\mathrm{I} 4)^{*}$ & --- \\
\hline \multicolumn{5}{|l|}{ Portulacaceae } \\
\hline Portulaca tuberosa Roxb. & $\mathrm{H}$ & $\mathrm{P}$ & $\mathrm{AM}^{*}(\mathrm{~A})^{*}, \mathrm{DSE}^{*}$ & --- \\
\hline \multicolumn{5}{|l|}{ Pteridaceae } \\
\hline Adiantum caudatum $\mathrm{L}$. & $\mathrm{H}$ & $\mathrm{P}$ & $\mathrm{AM}(\mathrm{I} 2)^{*}, \mathrm{DSE}^{*}$ & $\mathrm{AM}^{14,32}$ \\
\hline Adiantum lunulatum Burm. f. & $\mathrm{H}$ & $\mathrm{P}$ & $\mathrm{AM}(\mathrm{I} 2), \mathrm{DSE}$ & $\mathrm{AM}^{7,14},(\mathrm{I} 4)+\mathrm{DSE}^{12}$ \\
\hline Cheilanthes swartzii Webb \& Berthel. & $\mathrm{H}$ & $\mathrm{P}$ & $\mathrm{AM}(\mathrm{I} 2)$ & $\operatorname{AM}^{14,22}(\mathrm{I} 4)^{12}$ \\
\hline \multicolumn{5}{|l|}{ Rubiaceae } \\
\hline Mitracarpus villosus (Sw.) DC. & $\mathrm{H}$ & A & $\mathrm{AM}^{*}(\mathrm{I} 4)^{*}, \mathrm{DSE}^{*}$ & --- \\
\hline Oldenlandia herbacea (L.) Roxb. & $\mathrm{H}$ & $\mathrm{P}$ & $\mathrm{AM}^{*}(\mathrm{I} 4)^{*}, \mathrm{DSE}^{*}$ & --- \\
\hline \multicolumn{5}{|l|}{ Scrophulariaceae } \\
\hline Mecardonia procumbens (Mill.) Small & $\mathrm{H}$ & A & $\mathrm{AM}^{*}(\mathrm{~A})^{*}, \mathrm{DSE}^{*}$ & --- \\
\hline Striga asiatica (L.) Kuntze & $\mathrm{H}$ & A & $\mathrm{AM}^{*}(\mathrm{~A})^{*}, \mathrm{DSE}^{*}$ & NM $^{13,21}$ \\
\hline \multicolumn{5}{|l|}{ Selaginellaceae } \\
\hline Selaginella repanda (Desv. ex Poir.) Spring & $\mathrm{H}$ & $P$ & $\mathrm{AM}^{*}(\mathrm{I} 2)^{*}, \mathrm{DSE}^{*}$ & --- \\
\hline Selaginella vaginata Spring & $\mathrm{H}$ & $\mathrm{P}$ & $\mathrm{AM}^{*}(\mathrm{I} 4)^{*}, \mathrm{DSE}^{*}$ & --- \\
\hline \multicolumn{5}{|l|}{ Thelypteridaceae } \\
\hline Cyclosorus interruptus (Willd.) H.Itô & $\mathrm{H}$ & $\mathrm{P}$ & $\mathrm{AM}(\mathrm{I} 2)^{*}, \mathrm{DSE}^{*}$ & $\mathrm{AM}^{14}$ \\
\hline Macrothelypteris torresiana (Gaudich.) Ching & $\mathrm{H}$ & $\mathrm{P}$ & $\mathrm{AM}(\mathrm{I} 2)^{*}, \mathrm{DSE}^{*}$ & $\mathrm{AM}^{14,31}$ \\
\hline \multicolumn{5}{|l|}{ Urticaceae } \\
\hline Elatostema acuminatum (Poir.) Brongn. & US & P & $\mathrm{AM}^{*}(\mathrm{I} 4)^{*}, \mathrm{DSE}^{*}$ & --- \\
\hline Elatostema lineolatum Wight. & $\mathrm{H}$ & $\mathrm{P}$ & $\mathrm{AM}^{*}(\mathrm{I} 1)^{*}, \mathrm{DSE}^{*}$ & --- \\
\hline Girardinia diversifolia (Link) Friis & $\mathrm{H}$ & $\mathrm{P}$ & $\mathrm{AM}^{*}(\mathrm{I} 4)^{*}, \mathrm{DSE}^{*}$ & --- \\
\hline
\end{tabular}


Table 1. Cont.

\begin{tabular}{|c|c|c|c|c|}
\hline Family/Species & Life- form ${ }^{a}$ & Life cycle $^{b}$ & Fungal Association ${ }^{c}$ & Previous report $^{\mathrm{d}}$ \\
\hline Urticaceae & & & & \\
\hline Pouzolzia zeylanica (L.) Benn. & $\mathrm{H}$ & $\mathrm{P}$ & $\mathrm{AM}^{*}(\mathrm{I} 4)^{*}, \mathrm{DSE}^{*}$ & $\mathrm{NM}^{13,21}$ \\
\hline
\end{tabular}

${ }^{a} \mathrm{CH}$, Climbing herb; CS, Climbing shrub, H, Herb; S, Shrub; US, Under shrub; T, Tree

${ }^{b}$ A, Annual; P, Perennial

c,d NM, Non-mycorrhizal; AM morphological types: A, Arum, P, Paris; I1, intermediate-1; I2, intermediate-2; I4, intermediate-4

d Previous reports: ${ }^{1}$ Gemma et al. (1992), ${ }^{2}$ Harley \& Harley (1987a), ${ }^{3}$ Harley \& Harley (1987b), ${ }^{4}$ Hazarika et al. (2009), ${ }^{5} \mathrm{Hazarika}$ et al. (2010), ${ }^{6}$ Hemavani \& Thippeswamy (2013), ${ }^{7}$ Khade \& Rodrigues (2002), ${ }^{8}$ Kulkarni et al. (1997), ${ }^{9}$ Kumar \& Muthukumar (2014), ${ }^{10} \mathrm{Li}$ et al. (2004), ${ }^{11}$ Louis (1990), ${ }^{12}$ Muthukumar \& Prabha (2013), ${ }^{13}$ Muthukumar \& Udaiyan (2000a), ${ }^{14}$ Muthukumar \& Udaiyan (2000b), ${ }^{15}$ Muthukumar et al. (1996), ${ }^{16}$ Muthukumar et al. (2003), ${ }^{17}$ Muthukumar et al. (2004), ${ }^{18}$ Muthukumar et al. (2006), ${ }^{19}$ Muthuraja et al. (2014), ${ }^{20}$ Peyronel (1924), ${ }^{21}$ Ragupathy \& Mahadevan (1993), ${ }^{22}$ Raja et al. (1995), ${ }^{23}$ Santos et al. (2013), ${ }^{24}$ Songachan \& Kayang (2011), ${ }^{25}$ Sun et al. (2013), ${ }^{26}$ Tahira et al. (2012), ${ }^{27}$ Thapa et al. (2015), ${ }^{28} \mathrm{Uma}$ et al. (2010), ${ }^{29} \mathrm{Wang} \&$ Jiang (2015), ${ }^{30} \mathrm{Zhang}$ et al. (2011), ${ }^{31} \mathrm{Zhao}$ (2000), ${ }^{32}$ Zhao et al. (2006)

*New records

each plant species resulting in a total sample size of 216 . The roots were scooped off the rock surfaces, and the debris and mosses attached to roots were removed. The roots were then gently washed in water and fixed in FAA (formalinacetic acid-alcohol, $5 \mathrm{ml}: 5 \mathrm{ml}: 90 \mathrm{ml}$ ) and transported to the laboratory for processing. As most of the roots were not associated with soil, or identifiable spores were absent in the substrates attached to the roots, we did not enumerate AM fungal spore numbers or diversity.

\section{Preparation of roots for AM and DSE fungal assessment}

Fixed roots were washed free of FAA, cut into $1-\mathrm{cm}$ fragments, cleared in $10 \% \mathrm{KOH}$ (Phillips \& Hayman 1970), acidified with $5 \mathrm{~N} \mathrm{HCl}$ and stained with trypan blue $(0.5 \%$ in lacto glycerol) overnight. Roots that remained dark after clearing were bleached in alkaline $\mathrm{H}_{2} \mathrm{O}_{2}$ prior to acidification. The percentage of root length colonized by AM and DSE fungal structures and total root length colonized were estimated according to a magnified intersection method (McGonigle et al. 1990). Briefly, five intersections between the microscopic eyepiece crosshair and roots were recorded in $101-\mathrm{cm}$ long root bits for each plant at $\times 400$ in an Olympus BX5 trinolular microscope. The presence of AM and DSE fungal structures was noted at each intersections were recorded. The percentage of root length colonized by AM and DSE fungal structures and total root length colonized were estimated as the proportion of root length containing different fungal structures (McGonigle et al. 1990).

The AM-morphology was classified as Arum-, Parisand intermediate types based on whether the fungal hyphae were present, mainly as hyphae running through intercellular spaces or within cells as coils, respectively following descriptions of Dickson (2004). The intermediate subtypes categorized by Dickson (2004) are: linear hyphae are inter- and intracellular with the former possessing Arum- type arbuscules (intermediate type-1, I1); intracellular linear hyphae bearing Arum-type arbuscules (intermediate type2, I2); intracellular linear hyphae bearing arbuscules and intercellular linear hyphae (intermediate type-3, I3); hyphal/ arbusculate coils along with intercellular linear hyphae (intermediate type-4, I4). We checked the previous reports on AM and DSE fungal associations and AM colonization patterns, and the results are presented in Tab. 1 .

\section{Statistical analysis}

Analysis of variance (ANOVA) was used to assess the significance of variation for fungal variables among plant species. Similarly, ANOVA and $t$-test was used to test the influence of plant life-forms and lifecycle patterns on the fungal variables. Regression analysis was used to assess the relationship between AM and DSE fungal variables. Percentage data on AM and DSE fungal root colonization was arcsine transformed prior to analysis. All statistical analysis was performed using SPSS for Windows (Version 9).

\section{Results}

\section{Occurrence of AM and DSE fungal colonization}

In present investigation, the roots of 69 of the 72 plant species examined were AM and roots only three plant species (Murdannia nudiflora, Commelinaceae; Eriocaulon robustum, Eriocaulaceae; Drynaria quercifolia, Polypodiaceae), lacked AM fungal structures (Tab. 1). AM fungal colonization was characterized by the formation of a swollen appressorium on the root surface. The fungal hyphae originating from the appressorium spread inter and/or intracellularly as linear hyphae or hyphal coils to form various types of AM morphologies in the root cortex (Fig. 1A-K). Arbuscules were found on inter or intracellular linear hyphae or hyphal coils 

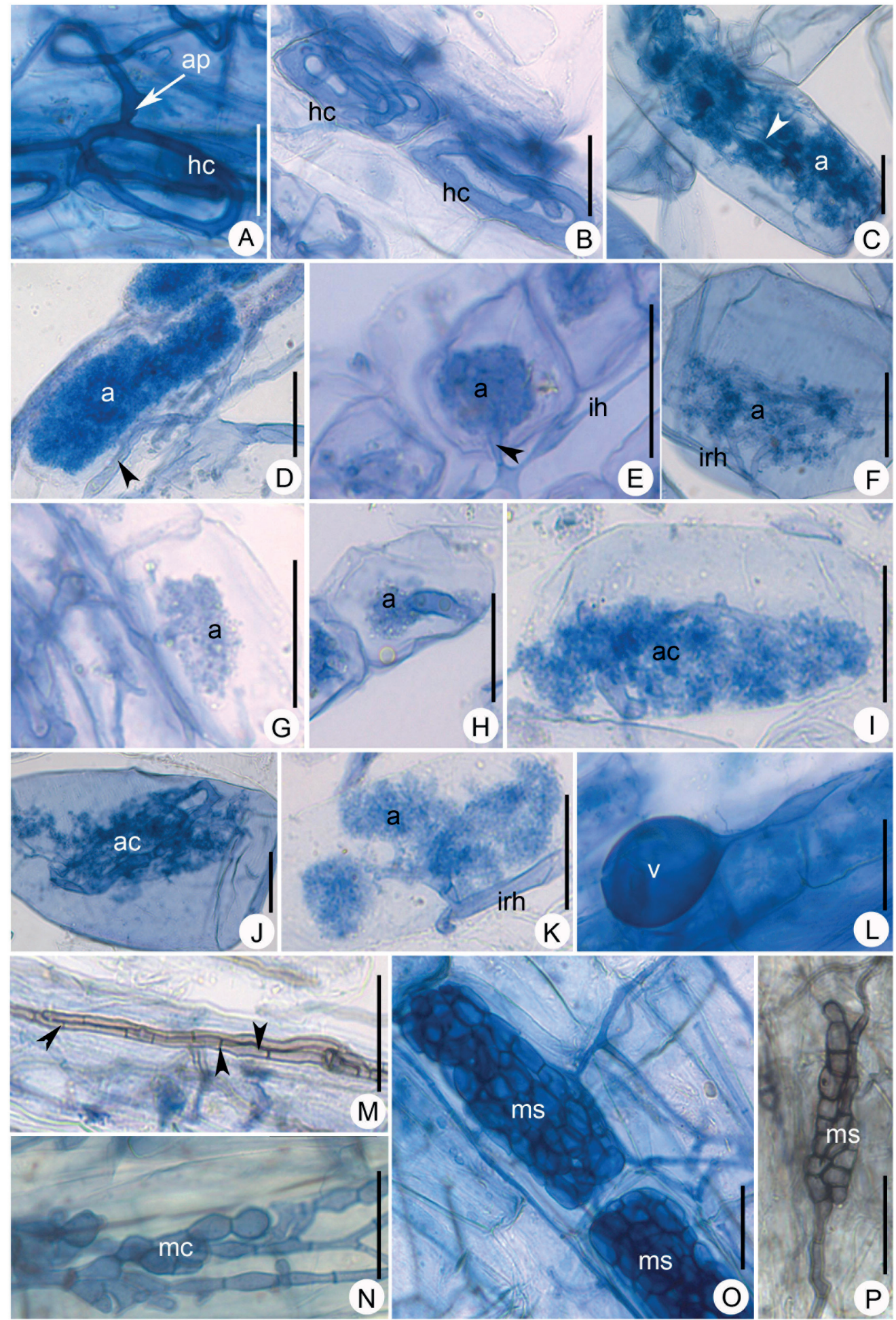

Figure 1. Arbuscular mycorrhizal (AM) (A-L) and dark septate endophyte (DSE) (M-P) fungi in the roots of lithophytic plant species of Siruvani Hills. (A) Appressorium (ap) and hyphal coil (hc) in the epidermal cell of Anredera baselloides, (B) Hyphal coils (hc) in cortical cells of Justicia diffusa, (C) Intracellular hyphae (white arrow head) and arbuscule (a) in Caralluma umbellata, (D) Intracellular hyphae (black arrow head) and arbuscule (a) in Hylodesmum repandum, (E) intercellular hyphae (ih), arbuscular trunk (black arrow head) and arbuscule (a) in Striga asiatica, (F) intracellular hyphae (irh) and arbuscule (a) in Lygodium flexuosum, (G-H) Arbuscule (a) in Anisochilus suffruticosus and Utricularia uliginosa, (I-J) Arbusculate coils (ac) in Biophytum sensitivum and Nephrolepis cordifolia, (K) Intracellular hyphae (irh) and arbuscule (a) in Strobilanthes asperrimus, (L) intercellular vesicle (v) in Acmella paniculata, (M) melanized septate hyphae (black arrow heads) in Eriocaulon robustum, $(\mathbf{N})$ moniliform cells (mc) in Eragrostis atrovirens, (O-P) microsclerotia $(\mathrm{ms})$ in Phaulopsis imbricata and Ageratina adenophora. Scale bars $=30 \mu \mathrm{m}$ 
(Fig. 1C-K). Vesicles were either inter and/or intracellular (Fig. 1L). Vesicles were absent in 13 plant species.

The majority of the plant species investigated (81\%) had DSE fungal colonization characterized by regularly septate hyaline/melanized hyphae bearing moniliform cells or microsclerotia (Fig. 1M-P). Of these, co-occurrence of DSE association along with AM was evident in $97 \%$ of these plant species. DSE fungal structures were absent in roots of Alstonia venenata (Apocynaceae), Argyreia populifolia, Merremia umbellata (Convolvulaceae), Costus speciosus (Costaceae), Agrostistachys borneensis, Phyllanthus urinaria (Euphorbiaceae), Smithia bigemina (Fabaceae), Parahemionitis cordata (Hemionietidaceae), Lygodium flexuosum (Lygodiaceae), Oplismenus burmanni, Setaria verticillata (Poaceae), Drynaria quercifolia, Pleopeltis macrocarpa (Polypodiaceae), Cheilanthes swartzii (Pteridaceae). Roots of two of the three nonmycorrhizal plant species (Murdannia nudiflora, Eriocaulon robustum) were colonized by DSE fungi (Tab. 1).

\section{Extent of AM fungal colonization}

The extent of AM fungal colonization and AM fungal structures varied significantly within plant species. The percentage root length with total colonization (\%RLTC) varied significantly among plant species $\left(\mathrm{F}_{71,144}=2.23\right.$; $\mathrm{P}<0.01$ ) and ranged from $17.45 \%$ (Phaulopsis imbricata, Acanthaceae) to $70.99 \%$ (Costus speciosus, Costaceae)
(Tab. 2). The percentage root length with hyphae (\%RLH) ranged from $2.70 \%$ in Cheilanthes swartzii (Pteridaceae) to $28.96 \%$ in Pleopeltis macrocarpa (Polypodiaceae). The \%RLH significantly varied among plant species $\left(\mathrm{F}_{71,144}=\right.$ 9.51; $\mathrm{P}<0.01)$. The percentage of root length with vesicles $(\% R L V)$ varied significantly among plant species $\left(\mathrm{F}_{71,144}=\right.$ 5.60; $\mathrm{P}<0.01$ ), and ranged from $1.93 \%$ in Crassocephalum crepidioides (Asteraceae) to $15.80 \%$ in Chamaecrista pumila (Fabaceae).

The percentage of root length with arbuscules (\%RLA, $\left.\mathrm{F}_{71,144}=44.49\right)$ and arbusculate coils (\%RLAC, $\mathrm{F}_{71,144}=$ 29.30; $\mathrm{P}<0.01)$ also significantly $(\mathrm{P}<0.01)$ varied with plant species. The \%RLA ranged from $7.82 \%$ in Phaulopsis imbricata (Acanthaceae) to $57.40 \%$ in Merremia umbellata (Convolvulaceae), and \%RLAC ranged from $8.02 \%$ in Oplismenus compositus (Poaceae) to $36.34 \%$ in Nephrolepis cordifolia (Nephrolepidaceae). The percentage of root length with hyphal coils (\%RLHC) ranged from 2.22\% (Nephrolepis cordifolia) to $30.37 \%$ (Blumea axillaris, Asteraceae) and significantly varied among plant species $\left(\mathrm{F}_{71,144}=8.57\right.$; $\mathrm{P}<0.01)$ (Tab. 2).

The average percentage of root length with different AM fungal structures (except \%RLA) and \%RLTC was maximum in trees and minimum in herbs (Fig. 2A). Among plants of different lifecycles, average \%RLH, \%RLA, \%RLV was higher in annuals than in perennials (Fig. 2B). Contrarily, perennials had a higher \%RLH, \%RLAC and \%RLTC than annuals. However, the differences between plant life-forms and life cycles were not significant.

Table 2. Extent of arbuscular mycorrhizal (AM) and dark septate endophyte (DSE) fungal colonization in non-orchidaceous lithophytes in Siruvani Hills of Western Ghats.

\begin{tabular}{|c|c|c|c|c|c|c|c|c|c|c|}
\hline \multirow{2}{*}{$\begin{array}{l}\text { Family } \\
\text { Species }\end{array}$} & \multicolumn{6}{|c|}{ AM colonization [\%] ${ }^{\mathrm{a}}$} & \multicolumn{4}{|c|}{ DSE colonization $[\%]^{\mathrm{b}}$} \\
\hline & RLH & RLHC & RLA & RLAC & RLV & RLTC & RLDH & RLMO & RLMI & RLDTC \\
\hline \multicolumn{11}{|l|}{ Acanthaceae } \\
\hline Phaulopsis imbricata & $9.63 \pm 0.55^{c}$ & --- & $7.82 \pm 0.56$ & --- & --- & $17.45 \pm 1.10$ & $23.50 \pm 1.07^{a}$ & $6.04 \pm 1.24$ & $6.04 \pm 1.24$ & $35.57 \pm 3.51$ \\
\hline Justicia diffusa & $24.27 \pm 3.94$ & $7.71 \pm 0.70$ & --- & $15.20 \pm 6.19$ & $4.12 \pm 0.52$ & $51.30 \pm 5.35$ & $14.17 \pm 0.81$ & --- & --- & $14.17 \pm 0.81$ \\
\hline Strobilanthes asperrimus & $15.08 \pm 1.46$ & -- & $32.89 \pm 0.44$ & --- & $4.60 \pm 0.64$ & $52.57 \pm 0.95$ & $10.52 \pm 0.62$ & $9.85 \pm 1.93$ & --- & $20.37 \pm 1.64$ \\
\hline \multicolumn{11}{|l|}{ Apocynaceae } \\
\hline Alstonia venenata & $18.37 \pm 2.69$ & $7.98 \pm 1.64$ & --- & $23.93 \pm 1.92$ & $3.06 \pm 0.61$ & $53.34 \pm 2.61$ & $4.90 \pm 0.60$ & $4.89 \pm 1.60$ & --- & $9.79 \pm 2.17$ \\
\hline \multicolumn{11}{|l|}{ Asclepidaceae } \\
\hline Caralluma umbellata & $7.08 \pm 0.40$ & -- & $31.00 \pm 4.00$ & -- & $3.10 \pm 0.58$ & $41.18 \pm 3.70$ & $7.65 \pm 2.38$ & $10.17 \pm 2.54$ & $1.96 \pm 0.00$ & $18.47 \pm 5.41$ \\
\hline \multicolumn{11}{|l|}{ Aspleniaceae } \\
\hline Asplenium zenkerianum & $20.65 \pm 3.48$ & --- & --- & $31.65 \pm 2.86$ & $9.68 \pm 3.47$ & $61.99 \pm 4.42$ & $10.39 \pm 0.73$ & --- & --- & $10.39 \pm 0.73$ \\
\hline \multicolumn{11}{|l|}{ Asteraceae } \\
\hline Acmella paniculata & $13.23 \pm 2.37$ & -- & $13.87 \pm 2.79$ & --- & $10.88 \pm 1.75$ & $37.97 \pm 2.68$ & $15.35 \pm 1.37$ & $12.36 \pm 1.25$ & $1.97 \pm 0.48$ & $29.69 \pm 1.42$ \\
\hline Ageratina adenophora & $10.79 \pm 0.47$ & --- & $9.91 \pm 0.72$ & --- & -- & $20.70 \pm 0.72$ & $6.61 \pm 0.86$ & $3.86 \pm 1.11$ & $1.58 \pm 0.00$ & $10.47 \pm 1.91$ \\
\hline Ageratum conyzoides & $22.97 \pm 3.84$ & $7.44 \pm 1.73$ & --- & $11.37 \pm 1.68$ & $6.11 \pm 2.80$ & $47.89 \pm 4.09$ & $12.38 \pm 2.38$ & $6.76 \pm 0.91$ & $2.23 \pm 0.50$ & $21.37 \pm 3.20$ \\
\hline Blumea axillaris & $11.10 \pm 3.39$ & $30.37 \pm 4.76$ & --- & $14.20 \pm 0.98$ & $3.58 \pm 1.10$ & $59.25 \pm 3.03$ & $11.11 \pm 4.52$ & $13.16 \pm 3.63$ & --- & $24.27 \pm 2.13$ \\
\hline Blumea oxyodonta & $19.41 \pm 2.18$ & $9.80 \pm 2.30$ & --- & $14.82 \pm 5.34$ & $6.86 \pm 0.15$ & $50.89 \pm 7.54$ & $15.37 \pm 3.36$ & --- & --- & $15.37 \pm 3.36$ \\
\hline Blumea virens & $7.70 \pm 1.14$ & $15.36 \pm 2.12$ & --- & $30.04 \pm 4.79$ & --- & $53.10 \pm 5.58$ & $6.43 \pm 2.80$ & $9.01 \pm 4.49$ & -- & $15.46 \pm 7.49$ \\
\hline Crassocephalum crepidioides & $25.13 \pm 4.18$ & --- & $17.35 \pm 3.14$ & -- & $1.93 \pm 0.03$ & $44.41 \pm 2.61$ & $6.43 \pm 0.55$ & $0.63 \pm 0.63$ & $2.90 \pm 0.83$ & $8.99 \pm 1.62$ \\
\hline Vernonia cinerea & $22.52 \pm 3.09$ & $5.95 \pm 1.07$ & --- & $18.47 \pm 1.59$ & $5.11 \pm 2.30$ & $52.05 \pm 1.60$ & $9.77 \pm 1.08$ & $9.12 \pm 2.79$ & --- & $18.90 \pm 3.45$ \\
\hline \multicolumn{11}{|l|}{ Balsaminaceae } \\
\hline Impatiens parvifolia & $18.41 \pm 1.68$ & -- & $16.98 \pm 0.74$ & --- & -- & $35.39 \pm 2.17$ & $9.10 \pm 1.96$ & $8.79 \pm 1.69$ & --- & $17.89 \pm 2.79$ \\
\hline \multicolumn{11}{|l|}{ Begoniaceae } \\
\hline Begonia malabarica & $20.74 \pm 3.10$ & $13.56 \pm 3.93$ & --- & $16.23 \pm 0.57$ & $5.17 \pm 0.59$ & $55.70 \pm 4.88$ & $12.38 \pm 1.89$ & $5.87 \pm 1.20$ & $3.17 \pm 0.60$ & $18.91 \pm 3.71$ \\
\hline \multicolumn{11}{|l|}{ Chenopodiaceae } \\
\hline Anredera baselloides & $5.37 \pm 1.09$ & -- & $15.72 \pm 0.91$ & --- & $9.81 \pm 3.33$ & $30.90 \pm 2.96$ & $13.74 \pm 5.64$ & $4.16 \pm 2.31$ & --- & $17.90 \pm 7.88$ \\
\hline
\end{tabular}




\section{Thangavelu Muthukumar, Marimuthu Chinnathambi and Perumalsamy Priyadharsini}

Table 2. Cont.

\begin{tabular}{|c|c|c|c|c|c|c|c|c|c|c|}
\hline \multirow{2}{*}{$\begin{array}{l}\text { Family } \\
\text { Commelinaceae }\end{array}$} & \multicolumn{6}{|c|}{ AM colonization [\%] } & \multicolumn{4}{|c|}{ DSE colonization [\%] $]^{b}$} \\
\hline & & & & & & & & & & \\
\hline Cyanotis cristata & $16.30 \pm 1.21$ & --- & $13.87 \pm 2.79$ & --- & $5.05 \pm 1.09$ & $35.22 \pm 4.12$ & $14.33 \pm 2.32$ & $5.62 \pm 1.58$ & $1.69 \pm 0.00$ & $20.51 \pm 4.15$ \\
\hline Murdannia nudiflora & --- & --- & --- & --- & --- & --- & $23.75 \pm 2.69$ & $3.27 \pm 1.04$ & --- & $27.02 \pm 2.98$ \\
\hline \multicolumn{11}{|l|}{ Convolvulaceae } \\
\hline Argyreia populifolia & $18.74 \pm 0.93$ & $13.80 \pm 1.03$ & --- & $23.70 \pm 1.09$ & $4.80 \pm 2.28$ & $61.03 \pm 1.05$ & --- & --- & --- & --- \\
\hline Merremia umbellata & $4.94 \pm 3.09$ & --- & $57.40 \pm 5.35$ & --- & --- & $62.34 \pm 6.17$ & --- & --- & --- & --- \\
\hline \multicolumn{11}{|l|}{ Costaceae } \\
\hline Costus speciosus & $19.88 \pm 2.49$ & $11.93 \pm 0.13$ & --- & $28.35 \pm 2.68$ & $10.84 \pm 2.57$ & $70.99 \pm 2.02$ & --- & -- & -- & -- \\
\hline \multicolumn{11}{|l|}{ Cyperaceae } \\
\hline Cyperus cyperinus & $9.92 \pm 0.73$ & --- & $33.49 \pm 2.89$ & --- & $3.45 \pm 0.99$ & $46.87 \pm 3.59$ & $13.31 \pm 2.17$ & $3.46 \pm 1.00$ & --- & $16.78 \pm 2.60$ \\
\hline \multicolumn{11}{|l|}{ Eriocaulaceae } \\
\hline Eriocaulon robustum & --- & --- & --- & --- & --- & -- & $24.90 \pm 1.60$ & $1.92 \pm 0.00$ & --- & $25.54 \pm 2.23$ \\
\hline \multicolumn{11}{|l|}{ Euphorbiaceae } \\
\hline Acalypha fruticosa & $22.49 \pm 2.03$ & $11.54 \pm 1.33$ & --- & $18.68 \pm 2.93$ & $5.46 \pm 1.04$ & $58.17 \pm 0.54$ & $8.50 \pm 0.69$ & --- & --- & $8.50 \pm 0.69$ \\
\hline Phyllanthus tenellus & $3.81 \pm 0.81$ & --- & $21.46 \pm 2.47$ & --- & $6.10 \pm 1.59$ & $31.37 \pm 4.46$ & $13.75 \pm 3.29$ & $4.25 \pm 1.79$ & -- & $18.00 \pm 2.46$ \\
\hline Phyllanthus urinaria & $18.15 \pm 0.70$ & --- & $21.42 \pm 0.93$ & --- & $10.10 \pm 1.52$ & $49.67 \pm 1.14$ & -- & --- & -- & --- \\
\hline \multicolumn{11}{|l|}{ Gentianaceae } \\
\hline Canscora heteroclita & $23.83 \pm 2.35$ & $17.13 \pm 2.37$ & --- & $19.75 \pm 0.62$ & $3.66 \pm 1.07$ & $64.36 \pm 5.32$ & $6.04 \pm 2.34$ & $1.82 \pm 0.03$ & -- & $7.25 \pm 2.68$ \\
\hline \multicolumn{11}{|l|}{ Gesneriaceae } \\
\hline Henckelia incana & $6.28 \pm 1.56$ & --- & $23.67 \pm 4.58$ & --- & $9.83 \pm 4.24$ & $39.77 \pm 7.52$ & $13.75 \pm 2.42$ & $6.08 \pm 0.86$ & --- & $19.82 \pm 2.84$ \\
\hline \multicolumn{11}{|l|}{ Fabaceae } \\
\hline Alysicarpus vaginalis & $17.19 \pm 1.87$ & --- & $17.40 \pm 2.10$ & --- & $12.43 \pm 0.40$ & $47.01 \pm 1.82$ & $11.94 \pm 2.29$ & $3.99 \pm 1.79$ & --- & $15.93 \pm 4.08$ \\
\hline Chamaecrista pumila & $24.94 \pm 1.46$ & $6.23 \pm 1.06$ & --- & $15.93 \pm 1.52$ & $15.80 \pm 1.59$ & $62.91 \pm 2.50$ & $7.43 \pm 0.83$ & $2.85 \pm 0.61$ & $3.63 \pm 0.00$ & $11.50 \pm 2.47$ \\
\hline Hylodesmum repandum & $9.57 \pm 0.42$ & --- & $28.28 \pm 2.27$ & --- & $8.48 \pm 2.59$ & $46.32 \pm 2.51$ & $20.93 \pm 3.16$ & $8.49 \pm 2.02$ & --- & $29.42 \pm 5.18$ \\
\hline Smithia bigemina & $19.04 \pm 3.01$ & $11.59 \pm 3.16$ & -- & $28.48 \pm 0.52$ & $10.57 \pm 2.98$ & $69.67 \pm 3.29$ & -- & -- & - & -- \\
\hline \multicolumn{11}{|l|}{ Hemionietidaceae } \\
\hline Parahemionitis cordata & $23.69 \pm 1.75$ & -- & $28.27 \pm 4.57$ & -- & -- & $51.96 \pm 3.70$ & -- & -- & -- & -- \\
\hline \multicolumn{11}{|l|}{ Lamiaceae } \\
\hline Pogostemon plectrantoides & $17.15 \pm 1.13$ & --- & $22.49 \pm 1.82$ & -- & $3.49 \pm 0.93$ & $43.13 \pm 1.17$ & $9.04 \pm 0.57$ & $6.75 \pm 1.65$ & $3.44 \pm 0.00$ & $19.23 \pm 2.22$ \\
\hline Lentibulariaceae & & & & & & & & & & \\
\hline Utricularia uliginosa & $11.30 \pm 0.53$ & --- & $20.49 \pm 3.28$ & -- & --- & $31.78 \pm 2.99$ & $10.79 \pm 1.34$ & $10.77 \pm 0.56$ & $1.69 \pm 0.00$ & $21.56 \pm 1.74$ \\
\hline Liliaceae & & & & & & & & & & \\
\hline Sansevieria zeylanica & $13.51 \pm 1.12$ & --- & $27.34 \pm 1.91$ & -- & $5.78 \pm 1.22$ & $46.63 \pm 0.96$ & $10.87 \pm 1.44$ & $1.96 \pm 0.00$ & -- & $12.18 \pm 1.91$ \\
\hline Lygodiaceae & & & & & & & & & & \\
\hline Lygodium flexuosum & $6.59 \pm 0.94$ & --- & $48.67 \pm 4.74$ & --- & $7.55 \pm 3.04$ & $62.81 \pm 1.93$ & --- & --- & -- & -- \\
\hline Malvaceae & & & & & & & & & & \\
\hline Hibiscus surattensis & $13.95 \pm 2.79$ & $24.83 \pm 5.62$ & --- & $21.35 \pm 4.65$ & $5.34 \pm 2.80$ & $65.47 \pm 8.28$ & $16.71 \pm 4.37$ & -- & -- & $16.71 \pm 4.37$ \\
\hline Melastomataceae & & & & & & & & & & \\
\hline Osbeckia wynaadensis & $3.96 \pm 0.68$ & --- & $25.90 \pm 5.30$ & --- & $8.67 \pm 3.86$ & $38.53 \pm 2.91$ & $9.97 \pm 2.60$ & $2.62 \pm 0.47$ & --- & $12.59 \pm 2.39$ \\
\hline Meliaceae & & & & & & & & & & \\
\hline Melia azedarach & $15.48 \pm 1.00$ & -- & $19.92 \pm 2.17$ & --- & --- & $35.40 \pm 1.23$ & $6.72 \pm 0.62$ & $3.05 \pm 0.82$ & --- & $10.30 \pm 0.46$ \\
\hline Moraceae & & & & & & & & & & \\
\hline Ficus microcarpa & $13.04 \pm 0.98$ & $6.73 \pm 2.05$ & -- & $19.30 \pm 1.78$ & $6.84 \pm 0.68$ & $45.90 \pm 1.16$ & $10.66 \pm 1.91$ & -- & -- & $10.66 \pm 1.91$ \\
\hline Nephrolepidaceae & & & & & & & & & & \\
\hline Nephrolepis cordifolia & --- & $2.22 \pm 0.53$ & --- & $36.34 \pm 0.97$ & $7.71 \pm 0.44$ & $46.27 \pm 0.45$ & $3.35 \pm 0.11$ & $2.81 \pm 0.60$ & --- & $6.16 \pm 0.69$ \\
\hline Oxalidaceae & & & & & & & & & & \\
\hline Biophytum sensitivum & $13.02 \pm 2.20$ & $4.61 \pm 0.70$ & --- & $22.85 \pm 1.59$ & --- & $40.48 \pm 2.40$ & $9.16 \pm 2.37$ & $5.22 \pm 1.70$ & $1.96 \pm 0.00$ & $15.03 \pm 1.31$ \\
\hline Poaceae & & & & & & & & & & \\
\hline Dimeria kanjirapalliana & $19.09 \pm 4.04$ & $9.21 \pm 1.88$ & -- & $17.56 \pm 3.31$ & $10.09 \pm 1.33$ & $55.95 \pm 1.52$ & $11.22 \pm 2.00$ & $7.41 \pm 1.83$ & -- & $18.64 \pm 1.57$ \\
\hline Eragrostis atrovirens & $9.26 \pm 2.35$ & $7.64 \pm 1.69$ & --- & $32.81 \pm 1.80$ & --- & $49.71 \pm 2.13$ & $12.22 \pm 1.57$ & $13.49 \pm 1.70$ & --- & $26.28 \pm 1.84$ \\
\hline Garnotia tenella & $15.06 \pm 2.96$ & $5.15 \pm 1.11$ & --- & $33.80 \pm 3.79$ & $9.02 \pm 1.04$ & $63.02 \pm 4.56$ & $13.75 \pm 3.28$ & $4.51 \pm 0.52$ & $2.26 \pm 0.59$ & $20.52 \pm 4.39$ \\
\hline Oplismenus burmanni & $6.57 \pm 0.63$ & $10.52 \pm 1.72$ & --- & $16.45 \pm 3.48$ & --- & $33.55 \pm 1.15$ & --- & -- & --- & -- \\
\hline Oplismenus compositus & $21.51 \pm 5.14$ & $6.79 \pm 0.62$ & --- & $8.02 \pm 0.62$ & $4.96 \pm 1.65$ & $41.28 \pm 5.18$ & $20.37 \pm 3.71$ & $1.85 \pm 0.02$ & $3.71 \pm 1.10$ & $25.93 \pm 4.71$ \\
\hline Pogonatherum crinitum & $20.24 \pm 5.23$ & $12.80 \pm 1.89$ & -- & $12.10 \pm 1.26$ & $6.37 \pm 0.67$ & $51.51 \pm 3.27$ & $21.59 \pm 2.49$ & $8.32 \pm 1.39$ & --- & $29.91 \pm 3.88$ \\
\hline Setaria verticillata & $14.34 \pm 0.81$ & $10.80 \pm 2.10$ & --- & $23.95 \pm 3.52$ & --- & $49.09 \pm 5.08$ & --- & -- & --- & --- \\
\hline Polypodiaceae & & & & & & & & & & \\
\hline
\end{tabular}


Table 2. Cont.

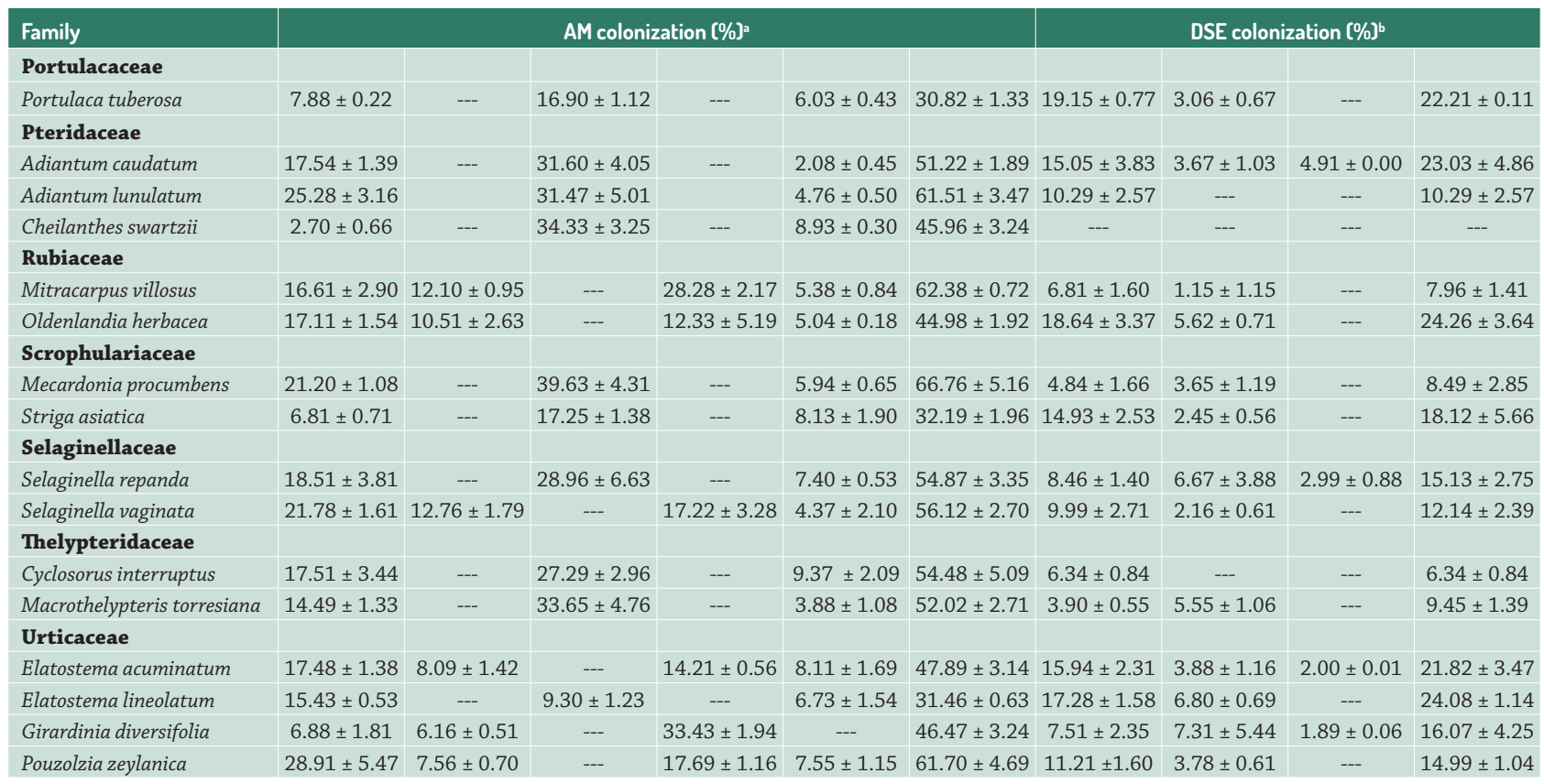

a RLH, RLHC, RLA, RLAC, RLV, RLTC, root length with hyphae, hyphal coils, arbuscules, arbusculate coils, vesicles, and total colonization respectively

${ }^{\mathbf{b}}$ RLDH, RLMO, RLMI, RLDTC, root length with dark septate fungal hyphae, moniliform cells, microsclerotia and total colonization respectively

${ }^{c}$ Mean \pm S.E.
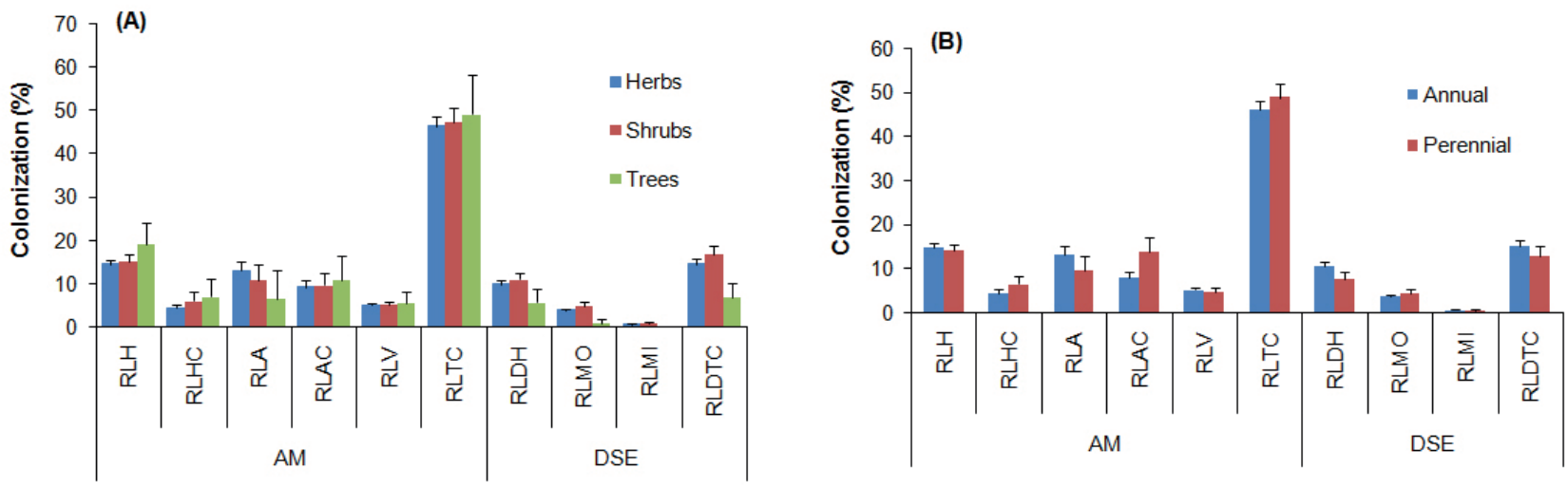

Figure 2. Average arbuscular mycorrhizal (AM) and dark septate endophyte (DSE) fungal colonization in lithophytes of various lifeforms (A) and life cycles (B). RLH, RLHC, RLA, RLAC, RLV, RLTC, root length with hyphae, hyphal coils, arbuscules, arbusculate coils, vesicles, and total colonization respectively. RLDH, RLMO, RLMI, RLDTC, root length with dark septate fungal hyphae, moniliform cells, microsclerotia and total colonization respectively. Error bars indicate \pm 1 SE.

\section{AM morphological types}

Intermediate type AM morphology was dominant among the various life-forms and lifecycles. Typical Paris-type AM was present only in the fern Nephrolepis cordifolia. Typical Arum-type AM was observed in 14 species and among the intermediate types; I1 was present in 10 species, I2 in 11 species and I4 in 33 species.

Among the various plant life-forms examined, $79 \%$ of the herbs, $71 \%$ of the under shrubs/shrubs and $100 \%$ of the trees had intermediate type AM (Fig. 3A). Arum-type AM was present in $<30 \%$ of the herbs and shrubs. Like plants in different life-forms, plants with different lifecycles were also dominated by intermediate type AM with $71 \%$ of the annuals and $76 \%$ of the perennials possessing it (Fig. 3B).

\section{Extent of DSE fungal colonization}

The percentage root length with DSE fungal colonization (\%RLDTC) ranged from $6.16 \%$ (Nephrolepis cordifolia) to 

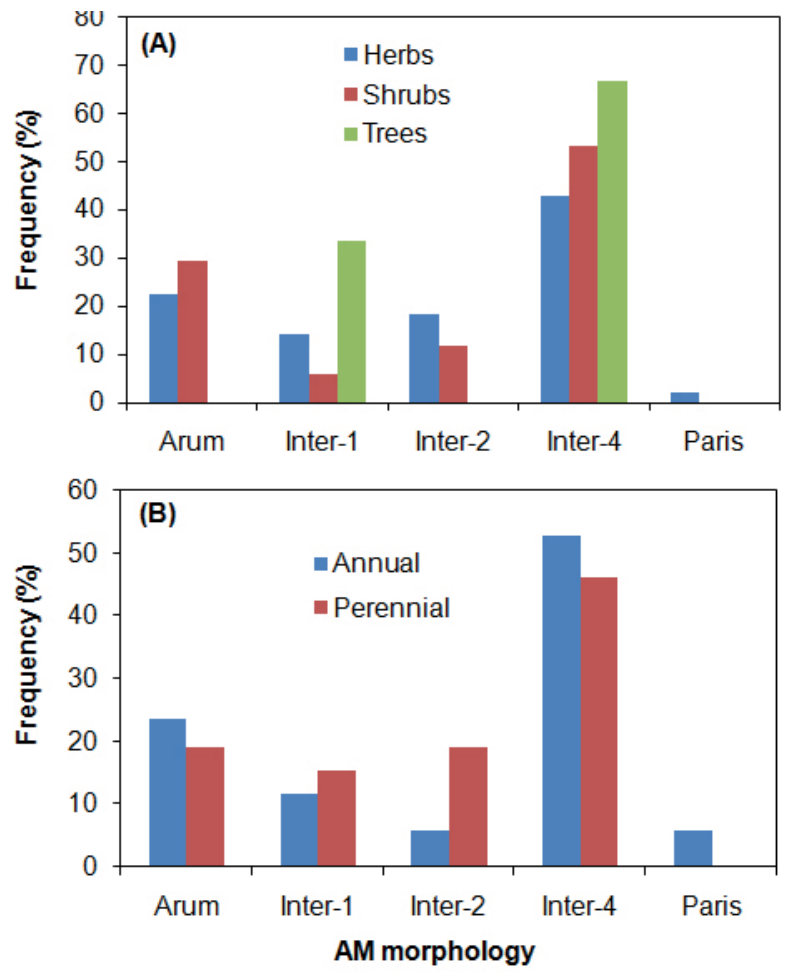

Figure 3. Frequency of arbuscular mycorrhizal (AM) morphology in lithophytes of various life-forms (A) and life cycle (B) patterns. Arum, Inter-1, Inter-2, Inter-4 and Paris represents Arum-type, intermediate type 1 , intermediate type 2 , intermediate type 4 and Paris-type respectively.

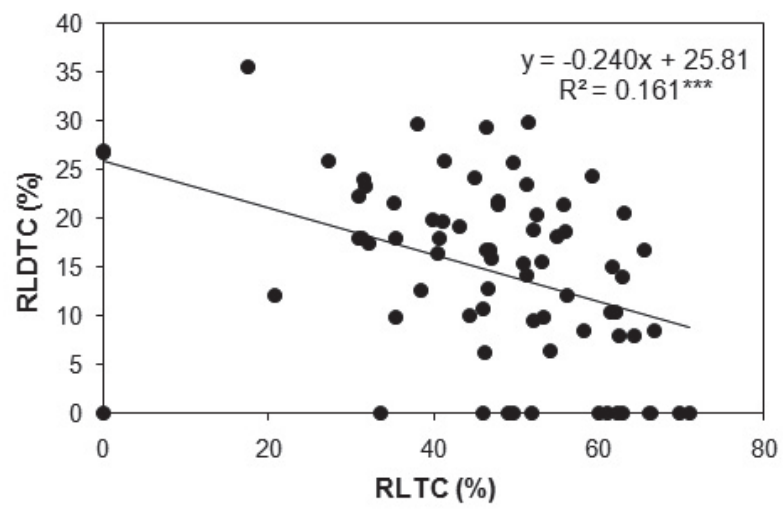

Figure 4. Relationship between total root lengths colonized by arbuscular mycorrhizal (RLTC) and dark septate endophytic (RLDTC) fungi in lithophytes. ${ }^{* * *}$ Significant at $\mathrm{P}<0.001$.

35.57\% (Phaulopsis imbricata, Acanthaceae). Root length with dark septate fungal hyphae (\%RLDH) ranged from $3.35 \%$ (Nephrolepis cordifolia) to $24.90 \%$ (Eriocaulon robustum, Eriocaulaceae) and root length with moniliform cells (\%RLMO) ranged from $0.63 \%$ (Crassocephalum cerpidioides, Asteraceae) to $13.49 \%$ (Eragrostis atrovirens, Poaceae) and microsclerotia (\%RLMI) ranged from $1.58 \%$ (Ageratina adenophora, Asteraceae) to $6.04 \%$ (Phaulopsis imbricata, Acanthaceae) (Tab. 2). DSE fungal structures like hyphae $\left(\mathrm{F}_{71,144}=8.11 ; \mathrm{P}<0.01\right)$, moniliform cells $\left(\mathrm{F}_{71,144}\right.$ $=5.75 ; \mathrm{P}<0.01)$, microsclerotia $\left(\mathrm{F}_{71,144}=3.58 ; \mathrm{P}<0.01\right)$ and total colonization $\left(\mathrm{F}_{71,144}=4.75 ; \mathrm{P}<0.01\right)$ varied significantly among species. Average \%RLDTC and average percentage root length with different DSE fungal structures were higher for shrubs than for trees and herbs (Fig. 2A). Likewise, average \%RLDH, \%RLMI and \%RLDTC were higher for annuals and average \%RLMO were higher for perennials (Fig. 2B). The \%RLDTC was significantly and negatively related to \%RLTC (Fig. 4)

\section{Discussion}

\section{Incidence of AM fungal colonization in lithophytes}

In this study, the lithophytic plant community consisted of species belonging to different plant groups and families. These results agree with the studies where lithophytic plant communities have been reported to contain a diverse plant assemblage (Gröger \& Huber 2007; Jayanthi et al. 2011). Ninety-six percent of the non-orchidaceous lithophytes examined in the present study was colonized by AM fungi and to our knowledge, AM symbiosis is reported for the first time in 42 plant species. High prevalence of AM fungal association in lithophytes in this study is in accordance with other studies where such an observation has been reported (Muthukumar \& Udaiyan 2000b; Zhang \& Guo 2007; Muthukumar et al. 2014; Muthuraja et al. 2014). Plants growing on rocks or shallow substrates employ several adaptations, including reduction or absence of root hairs and increasing the root diameter to maximize returns from limited carbon investments (Zwieniecki \& Newton 1995; Poot et al. 2012). This might have contributed to the high incidence of AM in lithophytes examined in the present study as coarse roots tend to increase the mycorrhizal dependency of plants (Fitter \& Moyersoen 1996).

The mycorrhizal status of some plant species appears to be significantly influenced by the nature of the substrate they grow. For example, Drynaria quercifolia reported mycorrhizal when growing on soil was nonmycorrhizal in the present study (Muthukumar et al. 2014; Muthuraja et al. 2014). This fern has been reported to be both mycorrhizal and nonmycorrhizal when growing as an epiphyte (Muthukumar \& Udaiyan 2000b; Muthukumar \& Prabha 2013). Like D. quercifolia, E. robustum examined was nonmycorrhizal. Species of Eriocaulon were reported to be both nonmycorrhizal (Ragupathy \& Mahadevan 1993; Muthukumar \& Udaiyan 2000a; Kumar \& Muthukumar 2014) and mycorrhizal (Khan \& Belik 1995; Brundrett et al. 1995; Rodrigues \& Bukhari 1995; Ragupathy et al. 1990; Harikumar 2001). Species in Eriocaulon have adaptations to cope with an extreme shortage of soil nutrients like $\mathrm{N}$ and P. In addition, plants of Eriocaulon are evergreen, and 
any nutrient investment in leaves is amortized over an extended period, which considerably reduces its reliance on soil resources and subsequently on AM association (Keddy 2010). Like other species of the genus, $M$. nudiflora was nonmycorrhizal in the present study (Logan et al. 1989; Brundrett et al. 1995; Tester et al. 1987; Silva et al. 2001; Muthukumar \& Udaiyan 2000a). However, non-functional mycorrhiza (lacking arbuscules) has been reported in Murdannia semiteres by Radhika \& Rodrigues (2007).

In contrast to earlier observations, Striga asiatica, the obligate root hemiparasite associated with grasses was colonized by both AM and DSE fungi (Ragupathy \& Mahadevan 1993; Muthukumar \& Udaiyan 2000a; Gworgwor \& Weber 2003). Nevertheless, atypical AM characterized by the presence of only hyphae and vesicles were reported in Striga gesnerioides parasitizing Lepidagathis hamiltoniana from the high-altitude region of Maharashtra, India (Kamble \& Agre 2013). Similarly, species like Chamaecrista pumila, Canscora heteroclita, Utricularia uliginosa, Lygodium flexuosum, Ficus microcarpa, Biophytum sensitivum, and Pouzolzia zeylanica previously reported to be nonmycorrhizal had AM fungal structures in the present study (Ragupathy \& Mahadevan 1993; Muthukumar \& Udaiyan 2000a; Khade \& Rodrigues 2002). This clearly emphasizes the need for more rigorous examination of plants from different habitats to resolve the true mycorrhizal status of a plant species (Wang \& Qiu 2006; Brundrett 2009). Habitat conditions like the availability of adequate AM fungal propagules or conditions necessary for the AM formation along with host factors contribute to the varied mycorrhizal status of a plant species (Smith \& Read 2008). Variations are likely to occur for facultatively mycorrhizal plant species that occupy diverse habitats (Brundrett 2009). Though the mycorrhizal status of sedges could be influenced by habitat conditions (Muthukumar et al. 2004),Cyperus cyperinus examined in the present study was mycorrhizal. This corresponds with the findings of Muthukumar et al. (1996) who also found AM in this sedge growing on soil. Results of recent experimental studies do indicate that sedges could benefit from AM association like other plant species (Lagrange et al. 2011; 2013).

Carnivorous plants inhabit nutrient-poor habitats and have highly specialized nutrient-capture strategies. These plants acquire substantial proportion of their nutrients by digesting their prey, and some have long and dense root hairs (Brundrett 2009; Ellison \& Adamec 2011). Subsequently, carnivorous plants have become partially or fully independent of mycorrhizal associations (Brundrett 2009). However, the roots of Utricularia uliginosa examined in the present study had both AM and DSE fungal structures. This is in accordance with studies where mycorrhizal structures have been reported in the roots of carnivorous plants (Fuchs \& Haselwandter 2004; Harikumar 2013). Although, the endophytic fungal associations have been reported in carnivorous plants, the nature and function of the associations have yet to be ascertained.

It is interesting to note that in spite of absence of spores in their substrate, more than $48 \%$ of lithophytes had $>50 \%$ of their roots colonized by AM fungi. This emphasizes the importance of propagules other than spores in initiating colonization under lithophytic conditions. The relevance of the extra radical mycelia and mycorrhizal roots as a propagule source in mycorrhization has already been well recognized (Schalamuk \& Cabello 2010).

\section{AM morphology}

We found the intermediate type AM to be common among lithophytes and even in different life-forms and lifecycles than typical Arum- and Paris-types. This is similar to some recent studies where intermediate type AM was found to be common among plant species examined. To our knowledge, we report AM morphology for the first time in 56 plant species. This complements Dickson et al. (2007) observation that even in plant species for which mycorrhizal status is available, AM morphologies is not known. Further, we also found AM morphologies different from those already reported in 12 plant species. This corroborates studies where different AM morphologies have been reported for the same plant species (see Dickson et al. 2007 and references therein). For example, Ageratum conyzoides possessing intermediate AM morphology in the present study is also capable of both Arum- and intermediate type morphologies (Muthukumar et al. 2003; Kumar \& Muthukumar 2014). Research had established that AM colonization patterns in plant roots are an interaction between plant root structure and the fungal partners. Although not examined in the present study, root structure, especially those of the cortical region tend to exhibit modifications in plants growing on rocks (Zwieniecki \& Newton 1995). This could have contributed to the variation in AM morphology in certain plant species from those already reported. Brundrett \& Kendrick (1990) indicated that the presence or absence of intercellular air spaces in the root cortical region to be an important determinant of AM morphology. Later studies elaborated this view and speculated the influence of factors like light, moisture and temperature of the substrates on AM patterns in roots as these factors can influence root growth (Yamato \& Iwasaki 2002; Yamato 2004; Becerra et al. 2007). However, detailed studies exploring root anatomy of lithophytic plant species, especially the intercellular spaces in roots could reveal their contribution to AM morphology. In addition to these, Cavagnaro et al. (2001) and Smith et al. (2004) have shown that AM fungal species colonizing roots could also influence AM fungal colonization patterns.

\section{Incidence of DSE fungal colonization in lithophytes}

Occurrence of DSE fungal association has been reported in a wide range of terricolous plant species belonging to 
diverse plant families (Mandyam \& Jumpponen 2015). Nevertheless, reports on the occurrence of DSE fungal association in non-terricolous plant species are limited. We found DSE fungal association in $82 \%$ of the plant species examined, which included Eriocaulon robustum and $M$. nudiflora the two of the three nonmycorrhizal species. The presence of DSE fungal association in lithophytes that lacked AM association resembles the observations of Lugo et al. (2009) in South American bromeliads where all the epiphytic nonmycorrhizal plants were invariably colonized by DSE fungi. Roots of Costus speciosus (Costaceae), Parahemionitis cordata and Drynaria quercifolia reported possessing DSE fungal association in earlier studies lacked DSE fungal structures in the present study (Uma et al. 2010; Muthukumar \& Prabha 2013; Muthuraja et al. 2014). To our knowledge, we report DSE fungal presence in 53 plant species for the first time. This clearly suggests the lack of information on the occurrence of DSE fungal occurrence in many habitats, especially in tropical regions (Jumpponen \& Trappe 1998). Based on their observations, Lugo et al. (2009) hypothesized that DSE fungi could enable plants to survive extreme arid conditions prevailing in nonterricolous habitats by enhancing the drought tolerance mechanisms and nutrient capture.

\section{Influence of plant habit and lifecycle on the occurrence of AM and DSE fungal associations}

In the present study, we did not find any significant difference in the extent of colonization for plants in different life-forms and lifecycles. This contrasts the observations of Muthukumar \& Udaiyan (2000a) where terricolous shrubs and trees growing in the Western Ghats region of southern India had higher colonization levels than herbs. Nevertheless, the average \%RLTC of $<50 \%$ in annuals and perennials accords with the observations of Muthukumar \& Udaiyan (2000a) where a higher proportion of annuals and perennials examined had colonization levels between $25 \%$ and $50 \%$. These clearly indicate that AM fungi are equally important for plant species with different life-forms and lifecycle strategies even under lithophytic conditions. Like AM fungal association, the extent of DSE fungal colonization also failed to exhibit significant variations among plants of different life-forms and lifecycle strategies. Nevertheless, the results of the present study suggest that lithophytes are more dependent on AM than DSE fungi. This is evidenced by the existence of a significantly higher average \%RLTC than \%RLDTC $\left(t_{71}=13.095 ; \mathrm{P}<0.001\right)$ in general as well as among different life-forms and lifecycles. For example, the \%RLTC in herbs, shrubs and trees was respectively 3.21, 2.82 and 7.30 folds higher than \%RLDTC. Similarly, the \%RLTC of annuals and perennials was 3.04 and 3.80 folds respectively higher than \%RLDTC. This may be due to more conducive conditions for AM formation and functioning and is consistent with the trend observed for terricolous species (Lugo et al. 2009; Muthukumar \& Prabha 2013).

\section{Relationship between AM and DSE fungi}

Co-occurrence of AM and DSE fungi were found in roots of $79 \%$ plant species examined. This is similar to studies where a high proportion of dual colonization of $\mathrm{AM}$ and DSE fungi in roots of plant species examined have been reported (Massenssini et al. 2014; Majewska et al. 2015). Our observation of a negative correlation between \%RLTC and \%RLDTC is in agreement with studies where an inverse relationship between these fungal variables has been reported (Muthukumar et al. 2006; Mandyam \& Jumpponen 2008; Muthukumar \& Tamilselvi 2010). While an interaction between AM and DSE fungal type is expected as they share the same niche within the root system, the nature of the relation between these two fungal types has received far less attention. It is generally believed that DSE fungi could aid plant growth and survival under circumstances where conditions are not conducive for AM functioning. For example, Barrow (2003) suggested that DSE fungi could enhance plant's performance in very dry soils of arid ecosystems. Similarly, DSE fungal colonization of the dominant grass Bouteloua gracilis from semi-arid grasslands of Mexico was four times higher than AM colonization (Medina-Roldán et al. 2008). The extent of root colonization by fungal endophytes depends on the carbon allocation by the host plant as well as the host, fungal and soil factors (Medina-Roldán et al. 2008; Smith \& Read 2008). Recent research on the control of plant-fungal mutualism indicates that the symbionts offering the best benefits in terms of nutrients or carbon are rewarded, and the control is bidirectional (Kiers et al. 2011; Hart et al. 2013). Therefore, differential allocation of carbon to these fungal types by host plants may reason for the inverse relation between these fungal variables in the present study.

\section{Conclusion}

Overall, the results of the present study indicate that $\mathrm{AM}$ and DSE fungal associations are widespread among plants growing on rocks like those of saxicolous plant species. This is the first report of AM and DSE fungal associations for several plant species. We speculate that the dual association of AM and DSE fungi in lithophytes could improve plant adaptability and survival under rapidly changing conditions of rock surfaces as shown for some harsh habitats. Despite their common occurrence, the roles of these fungal associations on plant adaptations to grow and survive on rock surfaces are not known unlike the role of bacteria (Puente et al. 2004a; b). Experimental studies are, therefore, necessary to ascertain the role of AM and DSE fungal associations on growth and nutrition of plants growing on rock surfaces. 


\section{References}

Alves R, Kolbeck J. 1993. Penumbral rock communities in Campo-Rupestre sites in Brazil. Journal of Vegetation Science 4: 357-366.

Balaji P, Hariharan GN. 2013. Checklist of microlichens in Bolampatti II forest range (Siruvani Hills), Western Ghats, Tamil Nadu, India. Czech Mycology 65: 219-232.

Barrow JR. 2003. Atypical morphology of dark septate fungal root endophytes of Bouteloua in arid southwestern U.S.A. rangelands. Mycorrhiza 13: 239-247.

Bashan Y, Khaosaad T, Salazar BG, et al. 2007. Mycorrhizal characterization of the boojum tree, Fouquieria columnaris, an endemic ancient tree from the Baja California Peninsula, Mexico. Trees Structure and Function 21: 329-335.

Bashan Y, Li CY, Lebsky VK, Moreno M, de-Bashan LE. 2002. Primary colonization of volcanic rocks by plants in arid Baja California, Mexico. Plant Biology 4: 392-402.

Becerra A, Cabello M, Chiarini F. 2007. Arbuscular mycorrhizal colonization of vascular plants from the Yungas forests, Argentina. Annals of Forest Science 64: 765-772.

Bledsoe C, Klein P, Bliss LC. 1990. A survey of mycorrhizal plants on Truelove Lowland, Devon Island, N.W.T., Canada. Canadian Journal of Botany 68: 1848-1856.

Brundrett M. 2009. Mycorrhizal associations and other means of nutrition of vascular plants: understanding the global diversity of host plants by resolving conflicting information and developing reliable means of diagnosis. Plant and Soil 320: 37-77.

Brundrett M, Ashwath N, Jasper D, et al. 1995. Mycorrhizal associations in the Alligator Rivers Region. Part II. Results of experiments. Final Report. Office of the Supervising Scientist, Jabiru N.T.

Brundrett M, Kendrick B. 1990. The roots and mycorrhizas of herbaceous woodlands plants. II. Structural aspects of morphology. New Phytologist 114: 469-479.

Cavagnaro TR, Gao LL, Smith FA, Smith SE. 2001.Morphology of arbuscular mycorrhizas as influenced by fungal identity. New Phytologist 151: 469-475.

Dickson S. 2004. The Arum-Paris continuum of mycorrhizal symbioses. New Phytologist 163: 187-200.

Dickson S, Smith FA, Smith S. 2007. Structural difference in arbuscular mycorrhizal symbioses: more than 100 years after Gallaud, where next? Mycorrhiza 17: 375-393.

Ellison AM, Adamec L. 2011. Ecophysiological traits of terrestrial and aquatic carnivorous plants: are the costs and benefits the same? Oikos 120: 1721-1731.

Fitter AH, Moyersoen B. 1996. Evolutionary trends in root-microbe symbioses. Philosophical Transactions of the Royal Society of London Series B 351: 1367-1375.

Fuchs B, Haselwandter K. 2004. Red list plants: colonization by arbuscular mycorrhizal fungi and dark septate endophytes. Mycorrhiza 14: 277-281.

Gemma JN, Koske RE, Flynn T. 1992. Mycorrhizae in Hawaiian Pteridophytes: occurrence and evolutionary significance. American Journal of Botany 79: 843-852.

Gold WG, Bliss LC. 1995. Water limitations and plant community development in a polar desert. Ecology 76: 1558-1568.

Gröger A, Huber O. 2007. Rock outcrop habitats in the Venezuelan Guayana lowlands: their main vegetation types and floristic components. Revista Brasileira de Botanica 30: 599-609.

Gworgwor NA, Weber HC. 2003. Arbuscular mycorrhizal fungi-parasitehost interaction for the control of Striga hermonthica (Del.) Benth. in sorghum [Sorghum bicolor (L.) Moench]. Mycorrhiza 13: 277-281.

Harikumar VS. 2001. Arbuscular mycorrhizal synthesis in some wetland plants in Kerala. Mycorrhiza News 12: 14-15.

Harikumar VS. 2013. Are there arbuscular mycorrhizal associations in carnivorous plants Drosera burmanii and D. indica? Botanica Serbica 37: 13-19.

Harley JL, Harley EL. 1987a. A check-list of mycorrhiza in the British flora. New Phytologist (Supplement) 105: 1-102.
Harley JL, Harley EL. 1987b. A check-list of mycorrhiza in the British flora-Addenda, errata and index. New Phytologist 107: 741-749.

Hart MM, Forsythe J, Oshowski B, Bücking H, Jansa J, Kiers ET. 2013. Hiding in a crowd--does diversity facilitate persistence of a low-quality fungal partner in the mycorrhizal symbiosis? Symbiosis 59: 47-56.

Hazarika S, Hazarika D, Barukial J. 2009. Arbuscular Mycorrhizal (AM) association with Ageratum conyzoides L., in relation to edaphic factors of Kaziranga Biosphere Reserve (KBR) Assam, India. International Journal of Plant Science 4: 259-261.

Hazarika S, Hazarika D, Barukial J. 2010. Arbuscular mycorrhizal association Ageratum conyzoides L. in different types of soil of Golaghat District, Assam, India. Advances in Plant Sciences 23: 153-154.

Hemavani C, Thippeswamy B. 2013. Arbuscular mycorrhizal fungi associated with some plants of Asteraceae in Bhadra wildlife sanctuary. International Journal of Plant and Environmental Sciences 3: 106-110.

Jayanthi P, Rajendran A, Thomas B, Aravindhan V, Sivalingam R. 2011. Biodiversity of lithophytes in Madukkarai Hills of Southern Western Ghats of Coimbatore District, Tamil Nadu, India. International Journal of Biological Technology 2: 76-82.

Jumpponen A, Trappe JM. 1998. Dark septate endophytes: a review of facultative biotrophic root colonizing fungi. New Phytologist 140: 295-310.

Kamble VR, Agre DG. 2013. New report on AMF colonization in root parasite Striga gesnerioides and its host Lepidagathis hamiltoniana from high altitude region of Maharashtra. International Multidisciplinary Research Journal 3: 27-31.

Keddy PA. 2010. Wetland ecology: Principles and conservation. Cambridge, Cambridge University Press.

Kessler M, Jonas R, Cicuzza D, et al. 2010. A survey of the mycorrhization of Southeast Asian ferns and lycophytes. Plant Biology 12: 788-793.

Khade SW, Rodrigues BF. 2002. Arbuscular mycorrhizal fungi associated with some pteridophytes from Western Ghat region of Goa. Tropical Ecology 43: 251-256.

Khan AG, Belik M. 1995. Occurrence and ecological significance of mycorrhizal symbioses in aquatic plants. In: Verma A, Hock B. (eds.) Mycorrhiza: structure, function, molecular biology and biotechnology. Heidelberg, Springer-Verlag. p. 627-666.

Kiers ET, Duhamel M, Beesetty Y, et al. 2011. Reciprocal rewards stabilize cooperation in the mycorrhizal symbiosis. Science 333: 880-882.

Kulkarni SS, Raviraja NS, Sridhar KR. 1997. Arbuscular mycorrhizal fungi of tropical sand dunes of west coast of India. Journal of Coastal Research 13: 931-936.

Kumar S, Muthukumar T. 2014. Arbuscular mycorrhizal and dark septate endophyte fungal associations in south Indian aquatic and wetland macrophytes. Journal of Botany. doi: 10.1155/2014/173125

Lagrange A, Ducousso M, Jourand P, Majorel C, Amir H. 2011. New insights into the mycorrhizal status of Cyperaceae from ultramafic soils in New Caledonia. Canadian Journal of Microbiology 57: 21-28.

Lagrange A, LHuillier L, Amir H. 2013. Mycorrhizal status of Cyperaceae from New Caledonian ultramafic soils: effects of phosphorus availability on arbuscular mycorrhizal colonization of Costularia comosa under field conditions. Mycorrhiza 23: 655-661.

Lehnert M, Kottke I, Setaro S, Pazmiño L, Lozano P, Kessler M. 2009. Mycorrhizal infections in ferns from southern Ecuador. American Fern Journal 99: 292-306.

Li T, Li J, Zhao Z. 2004. Arbuscular mycorrhizas in a valley-type savanna in southwest China. Mycorrhiza 14: 323-327.

Logan VS, Clarke PJ, Allaway WG. 1989. Mycorrhizas and root attributes of plants of coastal sand-dunes of New South Wales. Australian Journal of Plant Physiology 16: 141-146.

Lopez BR, Bashan Y, Bacilio M. 2009. Rock colonization plants: abundance of the endemic cactus Mammillaria fraileana related to rock type in the southern Sonoran Desert. Plant Ecology 201: 575-588.

Louis I. 1990. A mycorrhizal survey of plant species colonizing coastal reclaimed land in Singapore. Mycologia 82: 772-778.

Lugo MA, Molina MG, Crespo EM. 2009. Arbuscular mycorrhizas and dark septate endophytes in bromeliads from South American arid environment. Symbiosis 47: 17-21. 


\section{Thangavelu Muthukumar, Marimuthu Chinnathambi \\ and Perumalsamy Priyadharsini}

Majewska ML, Błaszkowski J, Nobis M, et al. 2015. Root-inhabiting fungi in alien plant species in relation to invasion status and soil chemical properties. Symbiosis 65: 101-115.

Mandyam K, Jumpponen A. 2005. Abundance and possible functions of the root-colonising dark septate endophytic fungi. Studies in Mycology 53: 173-190.

Mandyam K, Jumpponen A. 2008. Seasonal and temporal dynamics of arbuscular mycorrhizal and dark septate endophytic fungi in a tall grass prairie ecosystem are minimally affected by nitrogen enrichment. Mycorrhiza 18: 145-155.

Mandyam KG, Jumpponen A. 2015. Mutualism-parasitism paradigm synthesized from results of root-endophyte models. Frontiers in Microbiology 5: 1-13.

Massenssini AM, Bonduki VHA, Tótola MR, Ferreira FA, Costa MD. 2014. Arbuscular mycorrhizal associations and occurrence of dark septate endophytes in the roots of Brazilian weed plants. Mycorrhiza 24: 153-159.

McGonigle TP, Miller MH, Evans DG, Fairchild GL, Swan JA. 1990. A new method which gives an objective measure of colonization of roots by vesicular-arbuscular mycorrhizal fungi. New Phytologist 155: 495-501.

Medina-Roldán E, Arredondo J, Huber-Sannwald E, Chapa-Vargas L, Olalde-Portugal V. 2008. Grazing effects on fungal root symbionts and carbon and nitrogen storage in a short grass steppe in Central Mexico. Journal of Arid Environment 72: 546-556.

Muthukumar T, Prabha K. 2013. Arbuscular mycorrhizal and septate endophyte fungal associations in lycophytes and ferns of south India. Symbiosis 59: 15-33.

Muthukumar T, Tamilselvi V. 2010. Occurrence and morphology of endorhizal fungi in crop species. Tropical and Subtropical Agroecosystems 12: 593-604

Muthukumar T, Sathiyaraj G, Priyadharsini P, Uma E, Sathiyadash K. 2014. Arbuscular mycorrhizal and septate endophyte fungal associations in ferns and fern allies of Palni Hills, Western Ghats, southern India. Brazilian Journal of Botany 37: 561-581.

Muthukumar T, Senthilkumar M, Rajangam M, Udaiyan K. 2006. Arbuscular mycorrhizal morphology and dark septate fungal associations in medicinal and aromatic plants of Western Ghats, Southern India. Mycorrhiza 17: 11-24.

Muthukumar T, Udaiyan K. 2000a. Arbuscular mycorrhizas of plants growing in Western Ghats region, Southern India. Mycorrhiza 9: 297-313.

Muthukumar T, Udaiyan K. 2000b. Vesicular arbuscular mycorrhizae in pteridophytes of Western Ghats, Southern India. Phytomorphology 50: $132-142$.

Muthukumar T, Liqing S, Yang X, Cao M, Tang J, Zheng Z. 2003. Arbuscular mycorrhizae of plants in different vegetation types in Xishuangbanna, southwest China. Mycorrhiza 13: 289-297.

Muthukumar T, Udaiyan K, Manian S. 1996. Vesicular - arbuscular mycorrhizae in tropical sedges of Southern India. Biology and Fertility of Soils 22: 96-100.

Muthukumar T, Udaiyan K, Shanmughavel P. 2004. Mycorrhizae in sedgesan overview. Mycorrhiza 14: 65-77.

Muthuraja R, Muthukumar T, Sathiyadash K, Uma E, Priyadharsini P. 2014. Arbuscular mycorrhizal and dark septate endophyte fungal association in lycophytes and ferns of Kolli Hills, Eastern Ghats, Southern India. American Fern Journal 104: 67-102.

Newsham KK. 2011. A meta-analysis of plant responses to dark septate root endophytes. New Phytologist 190: 783-793.

Newsham KK, Upson R, Read DJ. 2009. Mycorrhizas and dark septate root endophytes in polar regions. Fungal Ecology 2: 10-20.

Peyronel B. 1924. Prime ricerche sulle micorrize endotrofiche e sulla microflora radicicola normale delle fanerogame. Rivistadi Biologia 6: 17-53.

Phillips JM, Hayman DS. 1970. Improved procedures for clearing roots and staining parasitic and vesicular-arbuscular mycorrhizal fungi for rapid assessment of infection. Transactions of the British Mycological Society 55: 158-161.

Poot P, Hopper SD, Diggelen JMH. 2012. Exploring rock fissures: does a specialized root morphology explain endemism on granite outcrops? Annals of Botany 110: 291-300.
Puente ME, Bashan Y, Li CY, Lebsky VK. 2004a. Microbial populations and activities in the rhizoplane of rock-weathering desert plants, I. Root colonization and weathering of igneous rocks. Plant Biology 6: 629-642.

Puente ME, Li CY, Bashan Y. 2004b. Microbial populations and activities in the rhizoplane of rock-weathering desert plants, II. Growth promotion of cactus seedlings. Plant Biology 6: 643-650.

Radhika KP, Rodrigues BF. 2007. Arbuscular mycorrhizae in association with aquatic and marshy plant species in Goa, India. Aquatic Botany 86: 291-294.

Ragupathy S, Mahadevan A. 1993. Distribution of vesicular arbuscular mycorrhizae in the plants and rhizosphere soils of the tropical plant, Tamil Nadu, India. Mycorrhiza 3: 123-136.

Ragupathy S, Mohankumar V, Mahadevan A. 1990. Occurrence of vesiculararbuscular mycorrhizae in tropical hydrophytes. Aquatic Botany 36: 287-291.

Raja P, Ragupathy S, Mahadevan A. 1995. Arbuscular mycorrhizal association of pteridophytes of Nilgiris and Kodaikanal hills, South India. Acta Botanica Indica 23: 181-186.

Read DJ, Haselwandter K. 1981. Observations on the mycorrhizal status of some alpine plant communities. New Phytologist 88: 341-352.

Rodrigues BF, Bukhari MJ. 1995. Occurrence of VAMF colonization in herbaceous plant species growing on iron ore mine wasteland in Goa. In: Reddy SM, Srivastava HP, Purohit DK, Reddy SR. (eds.) Microbial biotechnology. Jodhpur, Jodhpur University. p. 83-86.

Santos EA, Ferreira LR, Costa MD, Silva MCS, Reis MR, Maringá ACF. 2013. Occurrence of symbiotic fungi and rhizospheric phosphate solubilization in weeds. Acta Scientiarum Agronomy 35: 49-55.

Schalamuk S, Cabello M. 2010. Arbuscular mycorrhizal fungal propagules from tillage and no-tillage systems: possible effects on Glomeromycota diversity. Mycologia 102: 261-268.

Silva GA, Santos BA, Alves MV, Maia LC. 2001. Arbuscular mycorrhiza in species of Commelinidae (Liliopsida) in the state of Pernambuco (Brazil). Acta Botanica Brasilica 15: 155-165.

Smith SE, Read DJ. 2008. Mycorrhizal Symbiosis, 3rd. edn. New York, Academic Press.

Smith SE, Smith FA, Jakobsen I. 2004. Functional diversity in arbuscular mycorrhizal (AM) symbioses: the contribution of the mycorrhizal $\mathrm{P}$ uptake pathway is not correlated with mycorrhizal responses in growth or total P uptake. New Phytologist 162: 511-524.

Songachan LS, Kayang H. 2011. Diversity of arbuscular mycorrhizal fungi in pine forest of Meghalaya, North East India. Mycosphere 2: 497-505.

Sun X, Gao C, Guo LD. 2013. Changes in arbuscular mycorrhizal fungus community along an exotic plant Eupatorium adenophorum invasion in a Chinese secondary forest. Journal of Microbiology 51: 295-300.

Tahira JJ, Khan SN, Anwar W, Suliman R. 2012. Mycorrhizal association in some weeds of Curcuma longa fields of district Kasur, Pakistan. Pakistan Journal of Weed Science and Research 18: 331-335.

Tester M, Smith SE, Smith FA. 1987. The phenomenon of nonmycorrhizal plants. Canadian Journal of Botany 65: 419-431.

Thapa T, De UK, Chakraborty B. 2015. Association and root colonization of some medicinal plants with arbuscular mycorrhizal fungi. Journal of Medicinal Plants Studies 3: 25-35.

Tozer WC, Hackell D, Miers DB, Silvester WB. 2005. Extreme isotopic depletion of nitrogen in New Zealand lithophytes and epiphytes; the result of diffusive uptake of atmospheric ammonia? Oecologia 144: 628-635.

Uma E, Muthukumar T, Sathiyadash K, Muniappan V. 2010. Mycorrhizal and dark septate fungal associations in gingers and spiral gingers. Botany 88: 500-511.

Wang B, Qiu YL. 2006. Phylogenetic distribution and evolution of mycorrhizas in land plants. Mycorrhiza 16: 299-363.

Wang M, Jiang P. 2015. Colonization and diversity of AM fungi by morphological analysis on medicinal plants in southeast China. Scientific World Journal. doi:10.1155/2015/753842.

Yamato M. 2004. Morphological types of arbuscular mycorrhizal fungi in roots of weeds on vacant land. Mycorrhiza 14: 127-131.

Yamato M, Iwasaki M. 2002. Morphological types of arbuscular mycorrhizal fungi in roots of understory plants in Japanese deciduous broadleaved forests. Mycorrhiza 12: 291-296. 
Zhang Y, Guo L. 2007. Arbuscular mycorrhizal structures and fungi associated with mosses. Mycorrhiza 17: 319-325.

Zhang Y, Li T, Li L, Zhao ZW. 2011. The colonization of plants by dark septate endophytes (DSE) in the valley-type savanna of Yunnan, southwest China. African Journal of Microbiology Research 5: 55405547.
Zhao DD, Li LF, Zhao ZW. 2006. Three new records of arbuscular mycorrhizal fungi in China (in Chinese). Mycosystema 25: 142-144.

Zhao ZW. 2000. The arbuscular mycorrhizas of pteridophytes in Yunnan, southwestern China: evolutionary interpretations. Mycorrhiza 10: 145-149.

Zwieniecki MA, Newton M. 1995. Roots growing in rock fissures: their morphological adaptation. Plant and Soil 172: 181-187. 